\title{
Comparisons of lengths of lines separated horizontally and vertically
}

\author{
RICHARD A. GRIGGS and FRANK RESTLE \\ Indiana University, Bloomington, Indiana 47401
}

\begin{abstract}
Subjects made comparative ratings of two vertical lines. If the two lines were side by side, judgments were more accurate than if one line was higher in the field than the other. If one line was moderately higher in the field than the other, the higher line appeared shorter. This effect diminished when the higher line was raised, and disappeared when the higher line no longer overlapped with the lower. The direction of the illusion is opposite to the usual "anisotropy of visual space" or to expectations based on foreshortening or misplaced constancy. An explanation based on the configuration of the two lines, using the mathematical structure of adaptation level theory, was offered.
\end{abstract}

When a subject is asked to compare the lengths of two lines, as in many studies of psychophysics and perception. it is common procedure to have the two lines placed together in the visual field. The lines may be next to one another or separated. In the course of studies of illusions and other perceptual factors, we decided to study the subject's ability to compare the lengths of two vertical side-by-side lines as a function of how far apart they are, and whether one is higher in the field than the other. One simple hypothesis is that such comparisons are made by a process of mental manipulation (Metzler \& Shepard, 1974). If so, then performance should be better when the two lines are close together. since the necessary mental translation would be less. Also, performance should be better when the two lines are opposite one another, and worse when one is higher than the other. because of the vertical as well as horizontal component of mental movement required when one is above.

When one line is higher in the field than another. the discrepancy may not only affect accuracy but may introduce a constant error or illusion. For example, certain letters and numbers such as " $S$ " and " 8 " are usually printed with the upper part smaller. The difference in size between the upper and lower proportions usually goes unnoticed. However, if you invert them. this difference appears very large (Luckiesh. 1965; Stacey, 1969).

Koftkal (1935) concluded that perceived space has different properties in different directions, and is anisotropic. Stacey (1969) argued that a vertical line is perceived as pointing away in space. parallel to the viencer's line of sight. Therefore, the upper part of

This research was supported by United States Public Health Service Grant MH-16817 to Frank Restle. The authors would like to thank Jerry Forshee for programming assistance, and Conrad $G$. Mueller for valuable suggestions. Requests for reprints should be sent to Frank Restle, Department of Psychology. Indiana University. Bloomington. Indiana 47401. R. A. Griggs is now at the Liniversity of Florida. Gainesville. Florida. such a line is seen as foreshortened, and if the person compares two vertical lines of which one is higher in the field, he will see the higher as longer because of the greater foreshortening.

Piaget and Morf (1956) found that the top of two verticals was overestimated when two vertical lines were shown in extension. However, when the two vertical lines were shown with an increasing horizontal separation, the results were unclear; for some horizontal shifts. the upper vertical was underestimated. Piaget (1969) has argued that comparisons between vertical lines are made on the basis of centrations directed toward the tops of lines. In moving from one fixation point to another, more of the upper vertical is taken in and much of the lower rertical is excluded. On the basis of Piaget's law of centrations, that what is centered is also overestimated. it would follow that the upper vertical would be overestimated. However, the experimental results indicate that the illusion depends on the placement of the two lines. This suggests that the effects depend upon the overall configuration of the display.

One model for the process of comparing lines was recently suggested by Merryman and Restle (1970). These authors said that the comparison of two lines depended upon their judged length, where this judgment corresponds to the actual physical length divided by the adaptation level for length in the region of that line. An illusion, in such a theory, would result if two lines have different frames of reference of different length. Furthermore. Merryman and Restle salid that the difference between two lines can be thought of as the difference segment left over when the judgments of the two lines are subtracted. and that this difference is compared with the longer of the two lines.

This Merrman-Restle model divides the actual comparison between two lines into several stages. first. the subject may use the contiguration of the 
tigure to isolate crucial parts. The total configuration determines what line segments are used in comparative judgment. Second, adaptation levels for the various segments develop. and each subsegnent is judged with respect to its adaptation level. Third, these judged lengths are brought into correspondence so that the comparative judgment can be made.

In the Merryman-Restle formulation, if two segments, $S_{1}$ and $S_{2}$, are to be compared, first each is judged, yielding $J\left(S_{1}\right)$ and $J\left(S_{2}\right)$. Then the mean judgment of the difference is

$$
J(\text { Difference })=\frac{J\left(S_{1}\right)-J\left(S_{2}\right)}{\max \left[J\left(S_{1}\right), J\left(S_{2}\right)\right]} .
$$

Using the "adaptation-level" model,

$$
J\left(S_{1}\right)=S_{1} / A_{1},
$$

where $A_{1}$ is the "adaptation level" or weighted mean of lengths as centered at the location of Segment $S_{1}$.

According to such a formulation, having two lines side by side might help subjects make comparative judgments by setting up a configuration from which the difference between lines can be isolated by the subject. Constant errors would arise if for some reason the two segments being compared were in different contexts with different adaptation levels; that is, if $A_{1} \neq A_{2}$.

The present study measures the process of comparing the lengths of two lines when the lines are separated both horizontally and vertically. Carpenters, machinists, and other skilled workers must make such judgments with precision, and similar requirements are part of many ordinary activities. Though it appears that the placement of two lines has an important bearing on how well they can be compared, little is known either experimentally or theoretically about such comparisons. Such a study has implications not only for psychophysics and perception, but more broadly for cognitive psychology and the processes of imagination, mental manipulation, and judgment.

\section{METHOD}

\section{Subjects}

Thirty-two undergraduate students in introductory psychology at Indiana University served as subjects in partial fultillment of course requirements. Sixteen subjects served in each of two conditions.

\section{Materials}

Each stimulus presentation consisted of two vertical white (illuminated) lines on a dark background, projected on a $1.2 \mathrm{x}$ $1.2 \mathrm{~m}$ screen and viewed at a distance of $2 \mathrm{~m}$. The stand ard line was always $31 \mathrm{~cm}$ long. The other comparison line was of length 28.0 , 29.5 .32 .5 , or $34.0 \mathrm{~cm}$. Each line was approximately $1 \mathrm{~cm}$ in width, The comparison line was displaced upward from the standard a distance of $0,10.3 .20 .6,31.0$, or $41.3 \mathrm{~cm}$. (Note that $10.3 \mathrm{~cm}$ is one-third the length of the standard stimulus.) This displacement is measured from the bottom of the standard line vertically to the botton of the comparison line. Two sets of such figures were prepared. For Condition 1 , the standard and comparison lines were horizontally separated by $14 \mathrm{~cm}$, and in Condition ? by $6.5 \mathrm{~cm}$. The standard line was always in the center of the field, and the comparison was placed either to the right or to the left of the standard. Thus, in each condition, there were four lengths of comparison line, two positions left and right, and five vertical displacements of the comparison line, producing $4 \times 2 \times 5=40$ slides. In addition, there were 8 slides in which the comparison line was centered with the standard, having the same overlap above as below the standard. These 8 slides did not yield information needed in this study, and results are not reported below.

\section{Procedure}

Each slide was shown for $5 \mathrm{sec}$ or until all subjects had responded, whichever was shorter. The screen was dark for 3 sec between slides. Subjects viewed all 48 slides tive times in five different random permutations.

The subjects judged each pair of lines by pressing one of a row of six buttons. If the line on the right appeared longer, the subject was to press a button on the right. the outermost button corresponding to a judgment of "much longer," the intermediate button to "longer," and the centermost button to "slightly longer." If the left line appeared longer, the subject was to press a button on the left, again indicating the amount of difference by choosing one of the three buttons on the left. Subjects were instructed to respond as quickly, but as accurately, as possible. Response latencies were collected. All programming of stimuli. presentation of slides, and data collection used an IBM 1800 on-line computer.

\section{RESULTS}

The first replication was considered practice and ignored in all analyses.

A repeated measures analysis of variance revealed that there were no significant differences between 6.5. and $14-\mathrm{cm}$ spacing between test lines. Also, there were no significant differences between the four trials, and no significant differences between left and right placement of the comparison line. These three variables, therefore, were ignored in further calculations.

It was expected that if the two lines were side by side subjects might be more accurate in their judgments than if one line was higher than the other. This was measured by fitting a straight line to the function relating judgments to length of the comparison line. The steeper the slope of this line, the better discrimination the subject is displaying. Though the data do not perfectly fit a straight line, ${ }^{1}$ the fit is good enough to make the slope of a straight line an adequate index of over-all discriminability. The results of this calculation are shown in Table 1 . Line c. Notice that the discrimination is best when the two lines are even, and drops off sharply if one is traisslocated upward. It seems to make little difference whether the translocation is small (only one-third the length of the standard line) or quite large (four-thirds of the length of the standard line).

Fron this, it may be concluded, in accord with common sense. that if two lines are aligned their relative lengths can be distinguished by the excess of 
Table 1

Calculation of Constant Error as Function of Vertical Displacement

\begin{tabular}{|c|c|c|c|c|c|}
\hline & \multicolumn{5}{|c|}{ Vertical Displacement of Comparison Line } \\
\hline & .0 & 10.5 & 21.0 & 31.5 & 42.0 \\
\hline Mean judgment of comparison line (a) & 3.489 & 3.175 & 3.369 & 3.459 & 3.586 \\
\hline Mean constant error (in judgment units) (b) & -.011 & -.325 & -.131 & -.041 & +.086 \\
\hline Slope $\beta$ from $\mathbf{J}=\alpha+\beta \Phi(\mathrm{c})$ & .57 & .31 & .34 & .35 & .37 \\
\hline Inverse slope; $\mathrm{cm}$ as function of $\mathbf{J}(\mathrm{d})$ & 1.75 & 3.18 & 2.94 & 2.90 & 2.75 \\
\hline Mean constant error in $\mathrm{cm}: \mathrm{b} \times \mathrm{d}$ & -.02 & -1.03 & -.39 & -.12 & +.24 \\
\hline
\end{tabular}

one line over the other. Such comparisons can be judged accurately. If one line is higher in the field than the other. then each has an excess. one above and the other below, and the discrimination is more difficult to make.

A more interesting result. discovered unexpectedly, is a substantial constant error; the comparison line. when slightly higher in the field than the standard, is judged shorter than the standard. When the comparison is aligned with the standard, it is judged the same, and when it is very much higher than the standard. it is judged the same, but when the comparison is higher but overlapping with the standard. the comparison appears shorter.

The particular measure of this discrepancy depends on the fact that the experiment used comparative category responses (a comparison rating scale). Many psichophisical methods hold the response criterion constait (at. for example, a match in length of line) and vary the stimuli to find the point of subjective equality. Our rating-scale method prorides a fixed set of stimuli and lets the subject choose among a set of responses. To determine the apparent magnitude of an illusion. from category responses, one first determines the slope $(\beta)$ relating judgments to physical measurements (centimeters) within the configuration under question. Then one determines the magnitude of the raw illusory effect in the somewhat arbitrary judgment units. This effect in judgment units can then be translated into physical measurements by applying the slope $(1 / \beta)$. the inverse of the slope relating physical to judgment units Table 1 shows raw judgments. ${ }^{2}$ the discrepancy of this mean from the symmetrical response (which on a scale from 1 to 6 would be 3.5 ), the slope, the inverse slope for calculating physical illusions from judgments, and finally the mean constant error in centimeters. Notice that (1) there is almost no constant error when the two lines are aligned. (2) that there is a substantial constant error. with the comparison line judged shorter, when the comparison is slightly above the standard, and (3) that the effect crosses back over from negative to positive when the comparison line lies completely above the standard.

\section{DISCUSSION}

When two vertical lines are side by side and aligned. judgments of them are accurate and without constant error. When the comparison line lies slightly above. it looks shorter than the standard. When the comparison line is shifted still farther up, it steadily expands in apparent size until, when it lies completely above the standard. it begins to look slightly longer.

The result cannot be attributed to a response bias. since the experiment was completely symmetrical and the subjects could use the various response buttons with the same frequencies whether they did or did not display constant errors or illusions.

It is extremely unlikely that the result can be attributed to an "error of the standard" or any version of the "time-order effect error." The two lines are presented together and contemporaneously, and furthermore. in the procedure. the experimenter did not draw the subject's attention to one line or the other as a comparison line. In fact. there was no systematic way for an observer to know which line was the comparison line unless he could identify the $31-\mathrm{cm}$ line as distinguished from lines slightly longer or shorter. Only the comparison line was varied, but it appeared on the left and right equally often. Furthermore. the subject was not instructed to judge the standard or the comparison line, but merely to indicate which line of the two shown was longer and by how much.

Stacey's (1969) argument that the lines will be seen as extending away. so that the higher line is more foreshortened. is not in agreement even with the direction of our data. Another line of interpretation might appear to be that the two lines on the screen represent two fence posts or such similar vertical objects. If so, the line higher in the field would be thought of as farther away. Applying the principle of perceptual constancy (Gregory, 1963, 1966), the subject should therefore judge the higher comparison line to be longer. and this effect should tend to be greater the higher the line lies in the field. The opposite was observed. in that the higher comparison linte "las judged to be shorter rather than longer at a small displacement. and the effect then faded away as the line was moved still higher in the field.

The results may depend upon a process of mental translation or manipulation. However, the lateral separation of the two lines did not nake any difference. though wider spread lines would presumably require a greater translation, and would 


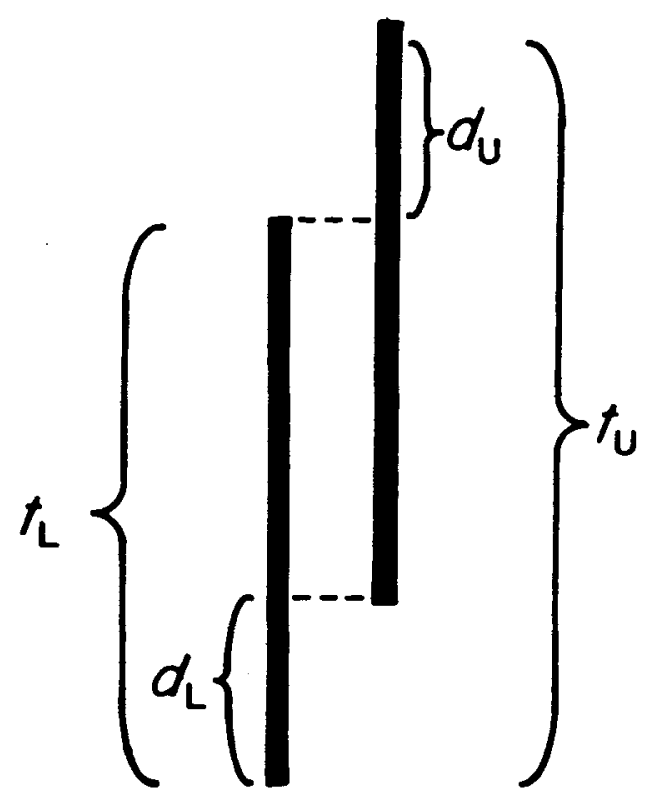

Figure 1. Comparison of two vertical lines when one is displaced upwards. The segments $d_{U}$ and $d_{L}$ are the differences between the two lines, upper and lower. The segments $t_{L}$ and $t_{U}$ are the total lines of which these differences are parts.

therefore be expected to reduce accuracy.

Since the effect is at a maximum when the comparison line is displaced upward one-third of the length of the standard, and decreases with greater displacement. the effect may probably depend upon the contiguration of two such lines. A possible explanation, based on the model of Merryman and Restle, can be constructed. The explanation assumes that when judging which of two overlapping lines is longer, the subject responds in terms of the parts of the two lines that hang over. extending beyond the position parallel to the end of the other line. Figure 1 shows a typical configuration in which the comparison line, on the right and higher than the standard, appears short. The segments $\mathrm{d}_{\mathrm{U}}$ and $\mathrm{d}_{\mathrm{L}}$ correspond to the overhanging parts at the upper and lower parts of the figure, respectively. If $\mathrm{d} U$ is judged smaller than $\mathrm{d}_{\mathrm{L}}$, then the comparison line will be judged shorter than the standard.

A special second hypothesis needed for this explanation is that in such a figure. the two lines are close enough to make a single contiguration. For some reason, perhaps based on experience, such a contiguration is interpreted as having a base across the botton of the configuration. Lengths of line segments are usually judged as discrepancies from this base level.

The explanation offered is this. Suppose that the tuo lines are actually the same length, so that $\mathrm{d} U$ is physically the same length as $d_{L}$. However, in judging du. the subject compares it in part with the distance from the top of the comparison line to the imaginary base line, a distance called $t U$. The segment $d_{L}$ is part of the segment $t_{L}$, which is, however, shorter than $t_{U}$. As at result. $d_{U}$ is judged shorter than $d_{L}$, even though it is physically equal, because $\mathrm{d} U$ has a somewhat larger immediate frame of reference.

This hypothesis correctly predicts the direction of the illusory effect. It also gives a sensible account of the fact that in the zero-displacement conditions, subjects show excellent discrimination. In that particular contiguration, $\mathrm{d}_{\mathrm{L}}$ is zero and the subject merely must discriminate $\mathrm{d} U$. In all other configurations, the subject must compare two extents that are somewhat separated in the field. Since $d_{L}$ is zero, there is no basis for a constant error in this configuration.

When the comparison line is displaced farther above the standard, the configuration changes. In particular, the segments $d_{U} U$ and $d_{L}$ become very large relative to the simple lengths of the lines, and therefore become less useful for making comparisons between the line lengths. Also, the standard and comparison lines get so separated that they are no longer seen as a configuration with a single base line. As the configuration changes, the particular comparison described above no longer holds, and as a result the illusory effect disappears.

This explanation, if correct. suggests that detailed studies of the perceptual and cognitive factors in psychophysical judgments, even comparisons of simple line lengths, may throw light on the effective perceptual performance shown in real-life situations.

\section{REFERENCES}

Gregory. R. L. Distortion of visual space as inappropriate constancy scaling. Nature. 1963,199, 678-680.

Gregory. R. L. Eye and brain. New York: World University Press, 1960.

KoffKa. K. Principle's of Gestalt psychology. New York: Harcourt. Brace, 1935.

Luckiesh. M. Visual illusions. New York: Dover, 1965. (Originally published by D. Van Nostrand, 1922.)

Merryman, C. T., \& Restle, F. Perceptual displacement of a test mark toward the larger of two visual objects. Journal of Experimental Psychology'. 1970, 84, 311-318.

MEtzler, J.. \& SHEPARD. R. N. Tiansformational studies of the internal representation of three-dimensional objects. In R. L. Solso (Ed.). Theories in cognitive psichology. Potomac, Md: Erlbaum. 1974.

Piaget, J. The mechanisms of perception (Trans. G. N. Seagrim). New York: Basic Books, 1969.

Piaget, J., \& Morf, A. Les comparisons verticales à taible intervalle. Archives de Psychologie, 1956, 35, 289-319.

StACEY, B. G. Explanations of the horizontal vertical illusion and the foreshortening of receding horizontals. Life Sciences. $1969,8,1237-1246$.

\section{NOTES}

1. In the Merryman-Restle theory, this function is not a straight line but is ogival in shape. The straight-line approximation is not erroneous in a fashion that invalidates the conclusion about discriminability.

2. For this purpose, the buttons were labeled 1 to 6 so that 1 meant the comparison line appeared much shorter and 6 meant it appeared much longer than the standard.

(Received for publication August 19, 1974; accepted December 5, 1974.) 